\title{
Bending Shear Stress for Solid Beams of Arbitrary Non-Symmetrical Cross-Section
}

\author{
T. YAhiaOUi* \\ Institute of Aeronautics and Space Studies, University of Blida 1, BP 270 Blida 09000, Algeria
}

(Received November 10, 2018; in final form February 4, 2019)

\begin{abstract}
The aim of this work is to develop a new numerical calculation program to calculate the shear stress subjected to a shear force for the general case of deflected bending, and to develop in this context a new numerical computational program for the calculation of the shear stress distribution in an arbitrary complex cross-section, in light to determine the position, and the value of the maximum stress, and consequently the determination of the shape factor seen in their practical importance. The calculation is valid for any axis and not necessary for the two principal axis of inertia. The calculation of the geometric characteristics of the section is necessary in this case. The formulae is done by the calculation of the static moment of the cut-off portion of section, where the calculation is made by an evaluation of an integral of complex function. View the obtained of a complex function, the calculation is given numerically by the use of the high order Gauss-Legendre formulae. The validation of the results is made by the convergence of the numerical accurate results to those for a chosen section like the arbitrary triangle which the exact result can be calculated analytically by the use of the new formulae. In this case, the solution is seen when the relative error given by the quadrature approaches to zero. The application will be for unsymmetrical section, views their practical interests in engineering.
\end{abstract}

DOI: 10.12693/APhysPolA.135.501

PACS/topics: shear stress, unsymmetrical section, shear force, shape factor, static moment, geometric characteristics, band width of the upper part of the section, gauss Legendre formulae, error of computation

\section{Introduction}

The shear stress $\tau$ calculation due to the effect of the shear force $T$ is generally done with respect to the principal axes of inertia for the case of simple bending, given the existence of the calculation formula in the literature [1-10]. In this case, the section is oriented in such a way that the shear force is applied according to one of the principal axes of inertia [11-15].

When the shear force is applied along an axis which is not necessarily the principal axis of inertia, which is the case for the deflected bending theory for a majority of the structures forming a mechanical, civil or Aerospace construction [10], the calculation of the tangential stress presented in the literature is done first of all by the consideration of the projection of the shear force according to the two principal inertias [1-15].

In this case, it is necessary to search the direction of the two principal axes of inertia and to calculate the stress $\tau$ along these two axes using the formula for simple bending [1-15]. This computation method is rather complicated in view of the arbitrary orientation of the section with respect to these axes. The computational geometry becomes very complicated for the evaluation of the variation of the incoming static moments in the calculation formula.

Sometimes it is impossible to orient one of the main axes of inertia along the axis of application of the shear force for technical reasons of construction.

*corresponding author
The calculation of the shear stress directly relative to the axis in question is impossible, also given the lack of an appropriate formula valid in this case. For this reason, the authors use the projection of the shear force along the principal axes of inertia and deduce the vectorial sum of the stresses according to the two projections. For example, in Refs. [1-15], the authors used the universal formula designed for simple flexion with respect to the principal axes of inertia for symmetric sections. In Ref. [10], the authors developed a program of computation of the shear stress for wing profiles only of the symmetric forms. Then all authors and industrial constructors, and up to now, and without exception [1-15], use only symmetric sections and the theory of simple bending in their calculations and construction for the evaluation of shear stress, for reasons of lack of analytical formula applicable to the non-symmetrical sections with respect to any axes, or simply neglecting the calculation of the shear stress by considering a fairly large length of the beam with respect to the transverse dimension and considering in this case only the calculation of the stress due to the bending moment.

This work focuses to develop a new formula and numerical calculation program to compute the distribution of the shear stress for the general case of deflected bending for arbitrary axis for beams having arbitrary complex cross-section, in light to determine the value and the position of maximum stress and consequently the determination of the general shape factor, in order to calculate the maximum shear stress within the bending strength test for the beams of small or moderate lengths for arbitrary form of the cross-section in the general case of deflected bending. 
The comparison and validation of numerical results will be made with implementing the program for the given unsymmetrical section like an arbitrary triangle where the exact solution can be analytically calculated by the use of the new formulae.

\section{Mathematical formulation}

The following Fig. 1 shows the general diagram of the shear stress $\tau_{y}$ and the direct stress $\sigma$ under the effect of a vertical shear force $T_{y}$ and a bending moment $M_{x}$ in any cross-section in the case of the deflected bending. For the case of the application of $\left(M_{y}\right.$ and $T_{x}$ ), one can deduce by analogy the corresponding scheme.

Under the effect of moment bending $M_{x}$, the normal force of the upper part is calculated by the following relation:

$$
\mathrm{d} N^{*}=\sigma \mathrm{d} A^{*} .
$$

The integration of (1) gives

$$
N^{*}=\int_{A^{*}} \sigma \mathrm{d} A^{*}
$$

Since the application of $M_{x}$ is made with respect to any axis which is not necessarily a principal axis of inertia, the normal stress $\sigma$ is calculated by the following relation according to the deflection bending theory by $[1-5]$ :

$$
\sigma=\frac{M_{x} I_{y}}{I_{x} I_{y}-I_{x y}^{2}} y^{*}+\frac{M_{x} I_{x y}}{I_{x} I_{y}-I_{x y}^{2}} x^{*} .
$$

Replacing relation (3) in relation (2) and integrating the result along the section of the upper part, we obtain

$$
N^{*}=\frac{M_{x} I_{y}}{I_{x} I_{y}-I_{x y}^{2}} S_{x}^{*}+\frac{M_{x} I_{x y}}{I_{x} I_{y}-I_{x y}^{2}} S_{y}^{*},
$$

(a)
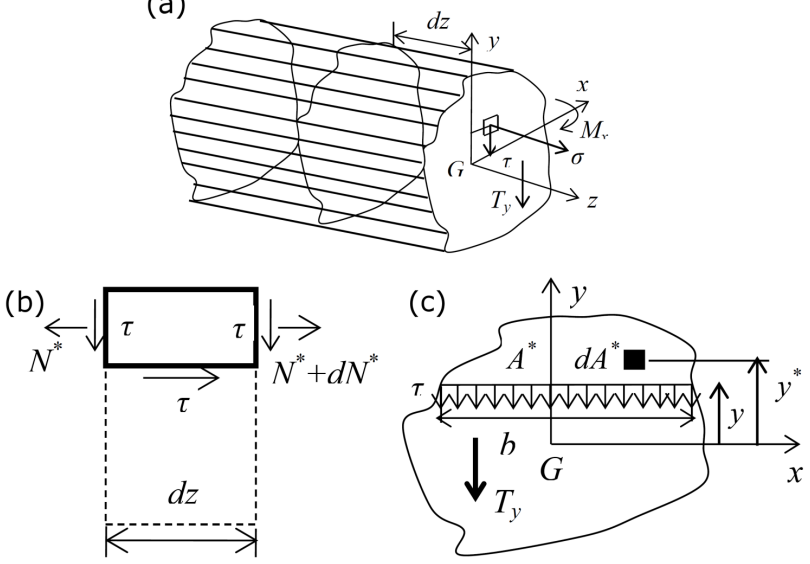

Fig. 1. Geometric presentation of shear stress due to shear force $T_{y}$ : (a) perspective view, (b) profile view, (c) front view. with

$$
S_{x}^{*}=\int_{A^{*}} y^{*} \mathrm{~d} A^{*}, S_{y}^{*}=\int_{A^{*}} x^{*} \mathrm{~d} A^{*} .
$$

The relation (4) contains two different terms. The first term depends on the geometry $\left(I_{x}, I_{y}, I_{x y}, S_{x}^{*}, S_{y}^{*}\right)$ of the section (which is given and unchanged), and the other term is the $M_{x}$ which depends on the internal forces. Then the differentiation of relation (4) affects only the internal forces and is given by the following relation:

$$
\mathrm{d} N^{*}=\mathrm{d} M_{x}\left(\frac{I_{y}}{I_{x} I_{y}-I_{x y}^{2}} S_{x}^{*}+\frac{I_{x y}}{I_{x} I_{y}-I_{x y}^{2}} S_{y}^{*}\right) .
$$

One can calculate the normal force by the consideration of the horizontal surface by intervening the shear stress $\tau$ by

$$
\mathrm{d} N^{*}=\tau b \mathrm{~d} z .
$$

Then the tangential stress $\tau_{y}$ can be calculated, starting from the relation (7), by

$$
\tau_{y}=\frac{d N^{*}}{b \mathrm{~d} z} .
$$

Replacing the relation (6) in relation (8), one obtains

$$
\tau_{y}=\frac{\mathrm{d} M_{x}}{b \mathrm{~d} z}\left(\frac{I_{y}}{I_{x} I_{y}-I_{x y}^{2}} S_{x}^{*}+\frac{I_{x y}}{I_{x} I_{y}-I_{x y}^{2}} S_{y}^{*}\right) .
$$

The derivative of the bending moment $M_{x}$ with respect to the longitudinal direction $z$ of the beam gives the value of the shear force along the $y$-axis by the following relation:

$$
\frac{\mathrm{d} M_{x}}{\mathrm{~d} z}=T_{y}
$$

setting

$$
Q=1-\frac{I_{x y}^{2}}{I_{x} I_{y}} .
$$

Replacing relations (10) and (11) in relation (9) we obtain the following relation:

$$
\frac{\tau_{y}}{T_{y}}=\frac{1}{b_{x} Q I_{x} I_{y}}\left(I_{y} S_{x}^{*}+I_{x y} S_{y}^{*}\right) .
$$

The relationship (12) gives the general formula for calculating the shear stress $\tau_{y}$ under the effect of a shear force $T_{y}$ applied along the arbitrary central $y$-axis for an arbitrary cross-section in the case of deflected bending.

For a section having one or two axes of symmetries, or with respect to the principal axes of inertia, the inertial product $I_{x y}$ with respect to these axes is equal to zero. The coefficient $Q$ given by the relation (11) becomes equal to unity. The relation (12) gives the famous calculation relation of the shear stress, applied for an axis of symmetry of a symmetrical section or with respect to the principal axes of inertia for any section [1-15]:

$$
\tau_{y}=\frac{T_{y} S_{x}^{*}}{b_{x} I_{x}} .
$$

By analogy and under the effect of the shear force $T_{x}$, the following relationship can be obtained directly from the relation (12) by changing $T_{y}$ by $T_{x}, \tau_{y}$ by $\tau_{x}, b_{x}$ by $b_{y}$, the $y$-axis by the $x$-axis, one obtains easily 


$$
\frac{\tau_{x}}{T_{x}}=\frac{1}{b_{y} Q I_{x} I_{y}}\left(I_{x} S_{y}^{*}+I_{x y} S_{x}^{*}\right) .
$$

The right member of the relations (12) and (14) depends only on the geometry of the section which is characterized by calculating the static moments $S_{x}^{*}$ and $S_{y}^{*}$ of the upper surface.

At the end, the position and the value of the stress $\tau_{\text {Max }}$ of the section can be determined.

The corresponding shape factor of the section can be calculated by the following relation:

$$
k=\frac{\tau_{\text {Max }}}{\tau_{\text {Average }}}=\frac{\tau_{\text {Max }}}{T} A .
$$

As we can see two shear forces $T_{x}$ and $T_{y}$, then we have two factors of form $k_{x}$ and $k_{y}$, respectively.

\section{Applications}

We present in this work three general forms of the sections determined with appropriate parameters. We are only interested in the section in the first quadrant.

Under the effect of $T_{y}$, the scheme shown in Fig. 1 can be considered. By analogy, the corresponding scheme can be deduced under the effect of $T_{x}$ from Fig. 1 .

\subsection{First section}

The general equation of this section is given by Eq. (16). We are only interested in the section in the first quadrant. Figure 2 shows the general shape of the sections chosen according to the parameters $n$ and $m$ illustrated in Table I:

$$
\left(\frac{x^{\prime}}{a}\right)^{n}+\left(\frac{y^{\prime}}{h}\right)^{m}=1 \text {. }
$$

Table I presents some sections studied according to parameters $n$ and $m$ and the curves in Fig. 2 .

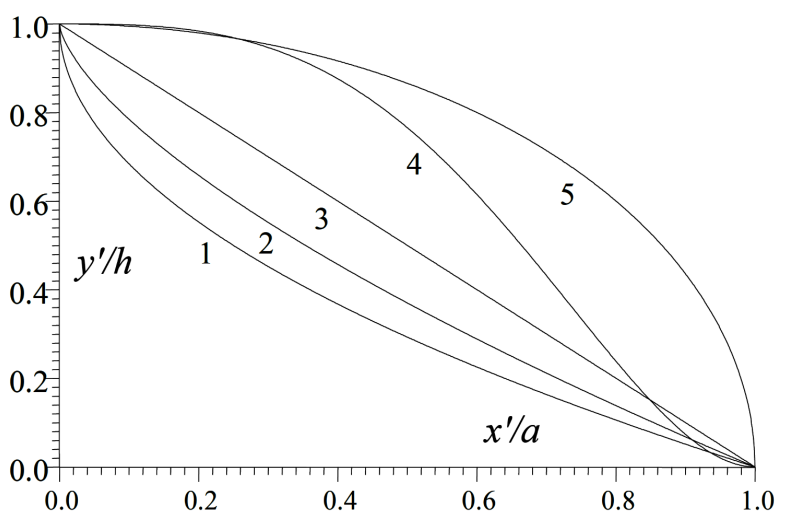

Fig. 2. Presentation of the shape of the five sections according to relation (16) and the parameters of Table I.

TABLE I

Some sections depending on the values of $n$ and $m$.

\begin{tabular}{c|c|c|c|c|c}
\hline \hline Curve & 1 & 2 & 3 & 4 & 5 \\
\hline$n$ & $2 / 3$ & $1 / 2$ & 1 & 2 & 3 \\
$m$ & 1 & 1 & 1 & 2 & $1 / 2$
\end{tabular}

Then, according to Fig. 2, curve 1 is for $n=2 / 3$, $m=1.0$. Curve 2 is for $n=1 / 2$ and $m=1.0$. Curve 3 is for $n=1.0$ and $m=1.0$. Curve 4 is for $n=2.0$ and $m=2.0$. Curve 5 is for $n=3.0$ and $m=1 / 2$.

To calculate the distribution of the shear stress by the formulae (12) and (14), it is necessary to calculate the geometrical characteristics of this section. Then we can prove the following formulae:

$$
\begin{aligned}
& A=a h \int_{0}^{1}\left(1-z^{n}\right)^{\frac{1}{m}} \mathrm{~d} z, \\
& x_{G}^{\prime}=\frac{a^{2} h}{A} \int_{0}^{1} z\left(1-z^{n}\right)^{\frac{1}{m}} \mathrm{~d} z, \\
& y_{G}^{\prime}=\frac{a h^{2}}{2 A} \int_{0}^{1}\left(1-z^{n}\right)^{\frac{2}{m}} \mathrm{~d} z, \\
& I_{x}=\frac{a h^{3}}{3} \int_{0}^{1}\left(1-z^{n}\right)^{\frac{3}{m}} \mathrm{~d} z-y_{G}^{\prime 2} A, \\
& I_{y}=a^{3} h \int_{0}^{1} z^{2}\left(1-z^{n}\right)^{\frac{1}{m}} \mathrm{~d} z-x^{\prime 2} A .
\end{aligned}
$$

For the calculation of the stress $\tau_{y}$, the static moments $S_{x}^{*}$ and $S_{y}^{*}$ as well as the length of the band $b_{x}$ of the upper part of Fig. 1 are calculated by the following relations:

$$
\begin{aligned}
& b_{x}=a\left[1-\left(\frac{y}{h}+\frac{y_{G}^{\prime}}{h}\right)^{m}\right]^{\frac{1}{n}}, \\
& S_{x}^{*}=a h^{2} \int_{y / h}^{1-y_{G}^{\prime} / h} z g \mathrm{~d} z, \\
& S_{y}^{*}=\frac{a^{2} h}{2} \int_{y / h}^{1-y_{G}^{\prime} / h} g\left(g-2 \frac{x_{G}^{\prime}}{a}\right] \mathrm{d} z,
\end{aligned}
$$

with

$$
g=\left[1-\left(z+\frac{y_{G}^{\prime}}{h}\right)^{m}\right]^{\frac{1}{n}}
$$

and

$$
-\frac{y_{G}^{\prime}}{h} \leq \frac{y}{h} \leq 1-\frac{y_{G}^{\prime}}{h} .
$$

For the computation of the stress $\tau_{x}$, the static moments $S_{x}^{*}$ and $S_{y}^{*}$, as well as the length of the right part of Fig. 3 are calculated by the following relations:

$$
\begin{aligned}
& b_{y}=h\left[1-\left(\frac{x}{a}+\frac{x_{G}^{\prime}}{a}\right)^{n}\right]^{\frac{1}{m}}, \\
& S_{y}^{*}=a^{2} h \int_{x / a}^{1-x_{G}^{\prime} / a} z g \mathrm{~d} z,
\end{aligned}
$$




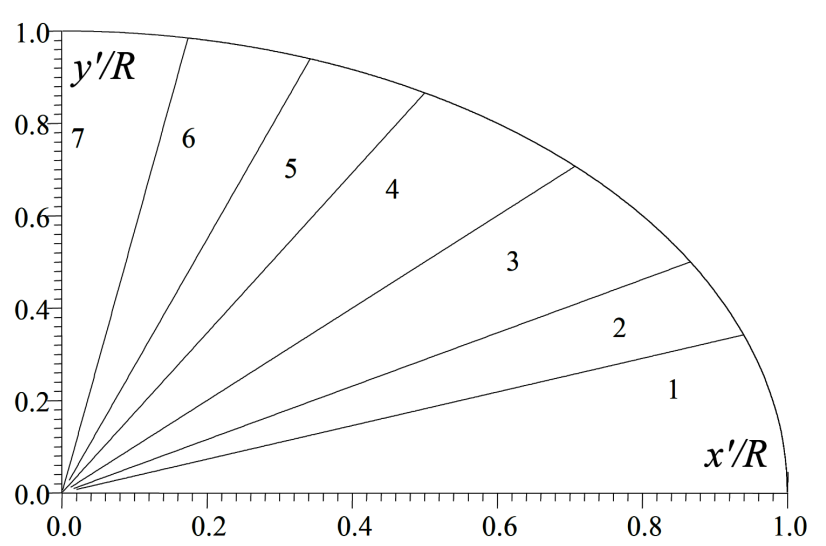

Fig. 3. Shape of the circular sector at angle $\theta$. Curve 1: $\theta=20^{\circ}$. Curve 2: $\theta=30^{\circ}$. Curve 3: $\theta=45^{\circ}$. Curve 4: $\theta=60^{\circ}$. Curve 5: $\theta=70^{\circ}$. Curve 6: $\theta=80^{\circ}$. Curve 7: $\theta=90^{\circ}$.

$$
S_{x}^{*}=\frac{a h^{2}}{2} \int_{x / a}^{1-x_{G}^{\prime} / a} g\left(g-2 \frac{y_{G}^{\prime}}{h}\right) \mathrm{d} z
$$

with

$$
g=\left[1-\left(z+\frac{x_{G}^{\prime}}{a}\right)^{n}\right]^{\frac{1}{m}}
$$

and

$$
-\frac{x_{G}^{\prime}}{a} \leq \frac{x}{a} \leq 1-\frac{x_{G}^{\prime}}{a} .
$$

For $n=m=1$, the relation (16) gives the equation of a triangle with a horizontal side equal to $a$ and the vertical side equal to $h$. In this case the integrals in the relations (17) to (31) can be evaluated analytically. The following results will be obtained:

$$
\begin{aligned}
& A=\frac{a h}{2}, \\
& x_{G}^{\prime}=\frac{a}{3}, \quad y_{G}^{\prime}=\frac{h}{3}, \\
& I_{x}=\frac{a h^{3}}{36}, \quad I_{y}=\frac{a^{3} h}{36}, \quad I_{x y}=-\frac{a^{2} h^{2}}{72} .
\end{aligned}
$$

Similarly, under the action of $T_{y}$ :

$$
\begin{aligned}
b_{x} & =a\left(\frac{2}{3}-\frac{y}{h}\right), \\
S_{x}^{*} & =\frac{a h^{2}}{3}\left(\frac{y}{h}+\frac{1}{3}\right)\left(\frac{2}{3}-\frac{y}{h}\right)^{2}, \\
S_{y}^{*} & =-\frac{a^{2} h}{6}\left(\frac{1}{3}+\frac{y}{h}\right)\left(\frac{2}{3}-\frac{y}{h}\right)^{2} .
\end{aligned}
$$

For the computation of the shear stress $\tau_{y}$ let us substitute the relations (22), (23), and (24) into the relations (12); we find after simplification the following results:

$$
\frac{\tau_{y}}{T_{y}}=\frac{20}{a h}\left(\frac{1}{3}+\frac{y}{h}\right)\left(\frac{2}{3}-\frac{y}{h}\right),
$$

$$
-\frac{1}{3} \leq \frac{y}{h} \leq \frac{2}{3}
$$

The position and the maximum value of the stress $\tau_{y}$ in relation (38) are

$$
\frac{\tau_{y_{\max }}}{T_{y}}=\frac{5}{a h} \text { for } \frac{y}{h}=\frac{1}{6} \text {. }
$$

Similarly, under the action of $T_{x}$, we shall have the following results:

$$
\begin{aligned}
b_{y} & =h\left(\frac{2}{3}-\frac{x}{a}\right), \\
S_{x}^{*} & =\frac{a^{2} h}{9}\left(3 \frac{x}{a}+1\right)\left(\frac{2}{3}-\frac{x}{a}\right)^{2}, \\
S_{y}^{*} & =-\frac{a h^{2}}{6}\left(\frac{1}{3}+\frac{x}{a}\right)\left(\frac{2}{3}-\frac{x}{a}\right)^{2} .
\end{aligned}
$$

Let us substitute relations (35), (36) and (37) in relations (14) we find after simplification the following result:

$$
\frac{\tau_{x}}{T_{x}}=\frac{20}{a h}\left(\frac{1}{3}+\frac{x}{a}\right)\left(\frac{2}{3}-\frac{x}{a}\right),
$$

with

$$
-\frac{1}{3} \leq \frac{x}{a} \leq \frac{2}{3}
$$

The position and maximum value of the stress $\tau_{y}$ in relation (23) are

$$
\frac{\tau_{x_{\max }}}{T_{x}}=\frac{5}{a h} \text { for } \frac{x}{a}=\frac{1}{6} .
$$

The shape factors of the triangular section can be calculated using Eq. (15) for the two shear forces along the axes $G_{x}$ and $G_{y}$ :

$$
k_{x}=k_{y}=\frac{5}{2} \text {. }
$$

For a symmetric triangular section (isosceles triangle), the variation of the shear stress as well as the value and position of the maximum stress and the shape factors are as follows [1-8]:

$$
\begin{aligned}
& \frac{\tau_{y}}{T_{y}}=\frac{12}{a h}\left(\frac{1}{3}+\frac{y}{h}\right)\left(\frac{2}{3}-\frac{y}{h}\right), \\
& \frac{\tau_{x}}{T_{x}}=\frac{12}{a h}\left(\frac{1}{3}+\frac{x}{a}\right)\left(\frac{2}{3}-\frac{x}{a}\right), \\
& \frac{\tau_{y \max }}{T_{y}}=\frac{\tau_{x_{\max }}}{T_{x}}=\frac{3}{a h} \text { for } \frac{x}{a}=\frac{y}{h}=\frac{1}{6},
\end{aligned}
$$

and

$$
k_{x}=k_{y}=\frac{3}{2} \text {. }
$$

According to results (47) and (51), the maximum stress with respect to the principal axis is $40 \%$ smaller than the maximum stress directed along the central axis of the definition of this section.

\subsection{Second section}

The shape of this second section represents a circular sector in the first quadrant limited between the horizontal axis and a straight line forming an angle $\theta$ with the 
horizontal as shown in Fig. 3. This section is defined by a radius $R$ and an angle of rotation $\theta$. When $\theta=90^{\circ}$, the circular sector becomes a quarter circle as the shape of the first section when $n=m=2.0$ (curve 4 ).

It can be shown that the geometric characteristics of this section can be calculated by the following formulae:

$$
\begin{aligned}
& A=\frac{\theta}{2} R^{2}, \\
& x_{G}^{\prime}=\frac{2 R}{3 \theta} \sin \theta, \\
& y_{G}^{\prime}=\frac{2 R}{\theta} \int_{0}^{\sin \theta} z\left(\sqrt{1-z^{2}}-\frac{z}{\tan \theta}\right) \mathrm{d} z, \\
& I_{x}=R^{4} \int_{0}^{\sin \theta} z^{2}\left(\sqrt{1-z^{2}}-\frac{z}{\tan \theta}\right) \mathrm{d} z-y^{\prime 2} A, \\
& I_{y}=\frac{R^{4}}{3} \int_{0}^{\sin \theta}\left[\left(1-z^{2}\right)^{\frac{3}{2}}-\left(\frac{z}{\tan \theta}\right)^{3}\right] \mathrm{d} z-x^{\prime 2} A, \\
& I_{x y}=\frac{R^{4}}{8} \sin ^{2} \theta-x_{G}^{\prime} y_{G}^{\prime} A .
\end{aligned}
$$

For the calculation of the stress $\tau_{y}$ by the relation (12), it can be demonstrated that the static moments $S_{x}^{*}$ and $S_{y}^{*}$ as well as the length of the bandwidth $b_{x}$ of the upper part of Fig. 1 can be calculated by the following relations:

$$
\begin{aligned}
& b_{x}= \\
& R\left[\sqrt{1-\left(\frac{y}{R}+\frac{y_{G}^{\prime}}{R}\right)^{2}}-\frac{1}{\tan \theta}\left(\frac{y}{R}+\frac{y_{G}^{\prime}}{R}\right)\right], \\
& S_{x}^{*}=R^{3} \int_{y / R}^{\sin (\theta)-y_{G}^{\prime} / R} z\left[\sqrt{1-g^{2}}-\frac{g}{\tan \theta}\right] \mathrm{d} z, \\
& S_{y}^{*}=\frac{R^{3}}{2} \int_{y / R}^{\sin \theta-y_{G}^{\prime} / R}\left[\left(-\frac{x^{\prime}{ }_{G}}{R}+\sqrt{1-g^{2}}\right)^{2}\right. \\
& \left.-\left(\frac{g}{\tan \theta}-\frac{x^{\prime}{ }^{2}}{R}\right)^{2}\right] \mathrm{d} z,
\end{aligned}
$$

with

$$
g=z+\frac{y_{G}^{\prime}}{R}
$$

and

$$
-\frac{y_{G}^{\prime}}{R} \leq \frac{y}{R} \leq \sin \theta-\frac{y_{G}^{\prime}}{R} .
$$

For the computation of the stress $\tau_{x}$ by the formula (14) it can also be shown that the static moments $S_{x}^{*}$ and $S_{y}^{*}$ as well as the length of the bandwidth $b_{y}$ of the upper part can be calculated by the following relations:

$$
\begin{aligned}
& b_{y}= \begin{cases}R\left(\frac{x}{R}+\frac{x_{G}^{\prime}}{R}\right) \tan \theta & \text { if }\left(\frac{x}{R}+\frac{x_{G}^{\prime}}{R}\right)<\cos \theta, \\
R \sqrt{1-\left(\frac{x}{R}+\frac{x_{G}^{\prime}}{R}\right)^{2}} \text { if }\left(\frac{x}{R}+\frac{x_{G}^{\prime}}{R}\right) \geq \cos \theta,\end{cases} \\
& S_{y}^{*}=R^{3} \int_{x / R}^{1-x_{G}^{\prime} / R} z g \mathrm{~d} z, \\
& S_{x}^{*}=\frac{R^{3}}{2} \int_{x / R}^{1-x_{G}^{\prime} / R} g\left(g-2 \frac{y_{G}^{\prime}}{h}\right) \mathrm{d} z
\end{aligned}
$$

with

$$
g= \begin{cases}\left(z+\frac{x_{G}^{\prime}}{R}\right) \tan \theta & \text { if }\left(z+\frac{x_{G}^{\prime}}{R}\right)<\cos \theta \\ \sqrt{1-\left(z+\frac{x_{G}^{\prime}}{R}\right)^{2}} & \text { if }\left(z+\frac{x_{G}^{\prime}}{R}\right) \geq \cos \theta\end{cases}
$$

and

$$
-\frac{x_{G}^{\prime}}{R} \leq \frac{x}{R} \leq 1-\frac{x_{G}^{\prime}}{R} .
$$

The results of the section of a quarter circle can be found in the first section for $n=m=2.0$ (curve 4) and in the second section when $\theta=90.0^{\circ}$ (curve 7 ). This gives us the possibility to validate the formulae found by the relations (17) to (31), and by the relations (52) to (68), and to confirm again the numerical calculation accuracy and the used quadrature.

\subsection{Third section}

The general equation of this section is given by Eq. (68). We are only interested in the section in the first quadrant. Figure 4 shows five shapes of five sections selected according to parameter $n$ illustrated in Table II:

$$
y^{\prime}=h\left(\frac{x^{\prime}}{a}\right)^{n},
$$

where $n$ is a non-zero positive real.

Table II presents five sections chosen according to the value of $n$. For $n=1$ we find the triangular section, where the results obtained must be the same as the first

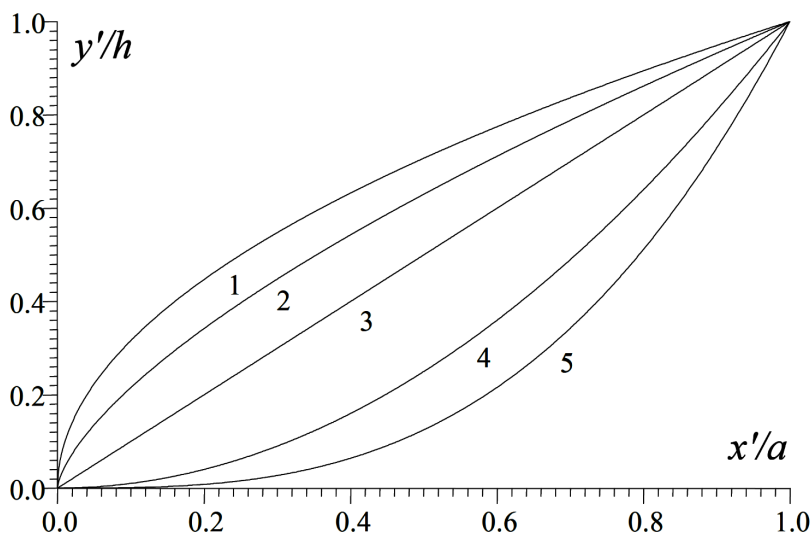

Fig. 4. Form of the third section according to the value of $n$. Curve 1: $n=2 / 3$. Curve 2: $n=1 / 2$. Curve 3: $n=1.0$. Curve $4: n=2.0$. Curve $5: n=3.0$. 
TABLE II

Some sections depending on the values of $n$ according to Fig. 4

\begin{tabular}{c|c|c|c|c|c}
\hline \hline Curve & 1 & 2 & 3 & 4 & 5 \\
\hline$n$ & $1 / 2$ & $2 / 3$ & 1 & 2 & 3
\end{tabular}

section when $n=m=1$ and with the analytical results presented by relations (35) to (47).

It can be shown that the geometric characteristics of this section can be calculated by the following formulae:

$$
\begin{aligned}
& A=\frac{a h}{n+1}, \\
& x_{G}^{\prime}=\frac{n+1}{n+2} a, \quad y_{G}^{\prime}=\frac{n+1}{2(2 n+1)} h, \\
& I_{x}=\frac{7 n^{2}+4 n+1}{12(3 n+1)(2 n+1)^{2}} a h^{3}, \\
& I_{y}=\frac{1}{(n+3)(n+2)^{2}} a^{3} h, \\
& I_{x y}=\frac{n}{4(n+1)(n+2)(2 n+1)} a^{2} h^{2} .
\end{aligned}
$$

For the calculation of the stress $\tau_{y}$ according to relation (12), the static moments $S_{x}^{*}$ and $S_{y}^{*}$, as well as the length of the bandwidth $b_{y}$ of the upper part of Fig. 1 can be calculated by the following relationships:

$$
\begin{aligned}
& b_{x}=a\left[1-\left(\frac{y}{h}+\frac{y_{G}^{\prime}}{h}\right)^{\frac{1}{n}}\right], \\
& S_{x}^{*}=a h^{2} \int_{y / h}^{1-y_{G}^{\prime} / h} z(1-g) \mathrm{d} z, \\
& S_{y}^{*}=\frac{a^{2} h}{2} \int_{y / h}^{1-y_{G}^{\prime} / h}\left[\left(1-\frac{x_{G}^{\prime}}{R}\right)^{2}-\left(g-\frac{x_{G}^{\prime}}{a}\right)^{2}\right] \mathrm{d} z,
\end{aligned}
$$

with

$$
g=\left(z+\frac{y_{G}^{\prime}}{h}\right)^{\frac{1}{n}}
$$

and

$$
-\frac{y_{G}^{\prime}}{h} \leq \frac{y}{h} \leq 1-\frac{y_{G}^{\prime}}{h} .
$$

For the calculation of the stress $\tau_{x}$ according to relation (14), the static moments $S_{x}^{*}$ and $S_{y}^{*}$, as well as the length of the bandwidth $b_{x}$ of the upper part of Fig. 1 can be calculated by the following relationships:

$$
\begin{aligned}
& b_{y}=h\left(\frac{x}{a}+\frac{x_{G}^{\prime}}{a}\right)^{n}, \\
& S_{y}^{*}=a^{2} h \int_{x / a}^{1-x_{G}^{\prime} / a} z g \mathrm{~d} z,
\end{aligned}
$$

$$
S_{x}^{*}=\frac{a h^{2}}{2} \int_{x / a}^{1-x_{G}^{\prime} / a} g\left(g-2 \frac{y_{G}^{\prime}}{h}\right) \mathrm{d} z,
$$

with

$$
g=\left(z+\frac{x_{G}^{\prime}}{a}\right)^{n}
$$

and

$$
-\frac{x_{G}^{\prime}}{a} \leq \frac{x}{a} \leq 1-\frac{x_{G}^{\prime}}{a} .
$$

\section{Calculation procedure}

Given the complexity of integral functions in relations (17) to (21), (23), (24), (28), (29), (54) to (56), (64), (65), (75), (76), (80) and (81), the analytical calculation is impossible. Hence, our interest is directed towards the determination of the numerical solution. In this context, the Gauss-Legendre quadrature of order 20 was used for regular functions [16-23]. This method compared with others methods, such as the rectangle, trapezium and the Simpson methods [16, 17], has a speed and convergence efficiency. It requires a very small number of points to have a high accuracy in comparison with the other methods; for example, for an accuracy of $10^{-8}$, only 20 Gauss points are needed. Therefore for rectangles, trapezius and Simpson we need, respectively about 1500000, 700000, and 200000 points. Then, the quadrature of the Gauss-Legendre is written as

$$
\int_{a}^{b} f(z) \mathrm{d} x=\frac{b-a}{2} \sum_{i=1}^{i=q} w_{i} f\left(z_{i}\right)
$$

with

$$
z_{i}=\frac{b+a}{2}+\frac{b-a}{2} \xi_{i} \quad(i=1,2,3, \ldots, q) .
$$

The values of $\xi_{i}$ and $w_{i}(i=1,2, \ldots, q)$ are, respectively the roots of the Legendre polynomial of order $q$ and the associated coefficients with the quadrature of Gauss-Legendre. For $q=20$, the values of $\xi_{i}$ and $w_{i}$ are shown in Table III.

TABLE III

Abscissas and coefficients of the Gauss-Legendre formulae of order 20 .

\begin{tabular}{c|c|c}
\hline \hline$i$ & $\xi_{i}$ & $w_{i}$ \\
\hline 1 & \pm 0.076526521133497333 & 0.152753387130725850 \\
2 & \pm 0.227785851141645078 & 0.149172986472603746 \\
3 & \pm 0.373706088715419560 & 0.142096109318382051 \\
4 & \pm 0.510867001950827098 & 0.131688638449176626 \\
5 & \pm 0.636053680726515025 & 0.118194531961518417 \\
6 & \pm 0.746331906460150792 & 0.101930119817240435 \\
7 & \pm 0.839116971822218823 & 0.083276741576704748 \\
8 & \pm 0.912234428251325905 & 0.062672048334109063 \\
9 & \pm 0.963971927277913791 & 0.040601429800386941 \\
10 & \pm 0.993128599185094924 & 0.017614007139152118
\end{tabular}


In relations (12) and (14), the stresses $\tau_{y}$ and $\tau_{x}$ depend respectively on the direction of the orientation of the cutting forces $T_{y}$ and $T_{x}$. Then for each axis one can calculate the said shear.

\section{Results and comments}

The results are quoted in three parts for the three selected sections. For each section, the variation of $S_{x}^{*}, S_{y}^{*}$, and $\tau_{y}$ is presented in the section when the shear force $T_{y}$ is applied and the variation of $S_{x}^{*}, S_{y}^{*}$ and $\tau_{x}$ when the shear force $T_{x}$ is applied followed by results tabulated on the geometric characteristics and the value and the position of the maximum stress and the associated shape coefficient.

Each figure contains 5 to 7 curves according to the value of the parameters defining the geometry of the section.

The variation of the parameters in Figs. 5 to 22 is presented in the reference of the definition of the section after making a change of reference between the central axis and the axis of definition of the section, that the variation of the static moments in the relationships (23), $(24),(28)$, and (29) for the first section, and in the relationships (59), (60), (64), and (65) for the second section and in the relations $(75),(76),(80)$, and (81) for the third section are presented in a central reference mark according to the relations (26), (31), (62), (67), (78), and (83), respectively.

All results are determined in a non-dimensional manner. All we did was simply change the variable to the benchmark of the section definition.

\subsection{First section}

Figures 5 to 10 contain 5 curves for 5 sections chosen according to the values of $n$ and $m$. Then curve 1 is for $n=2 / 3, m=1.0$. Curve 2 is for $n=1 / 2$ and $m=1.0$. Curve 3 is for $n=1.0$ and $m=1.0$. Curve 4 is for $n=2.0$ and $m=2.0$. Curve 5 is for $n=3.0$ and $m=1 / 2$.

Figure 2 also shows the variation in the length of the bandwidth $b_{x} / a$ as a function of the ordinate $y^{\prime} / h$ for

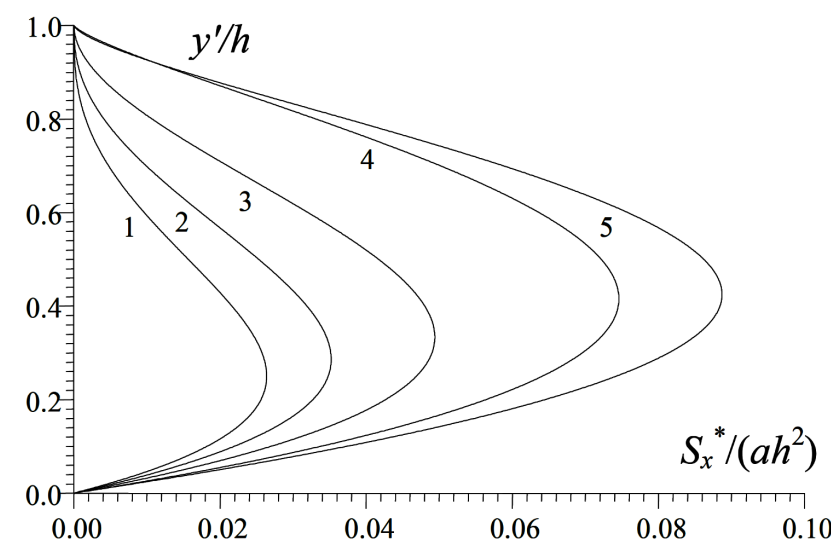

Fig. 5. Variation of static moment $S_{x}^{*} /\left(a h^{2}\right)$ as a function of the ordinate $y^{\prime} / h$ for some sections according to the values of $n$ and $m$ of Table I.

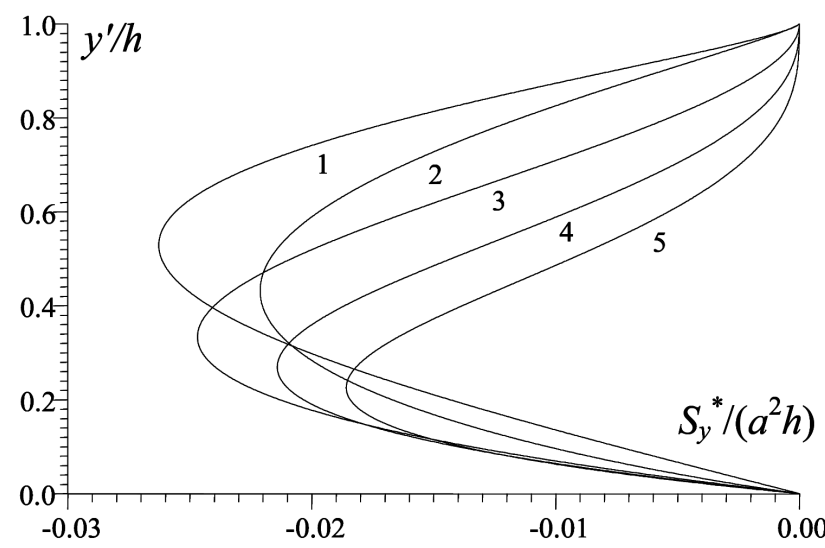

Fig. 6. Variation of static moment $S_{y}^{*} /\left(a^{2} h\right)$ as a function of the ordinate $y^{\prime} / h$ for some sections according to the values of $n$ and $m$ of Table I.

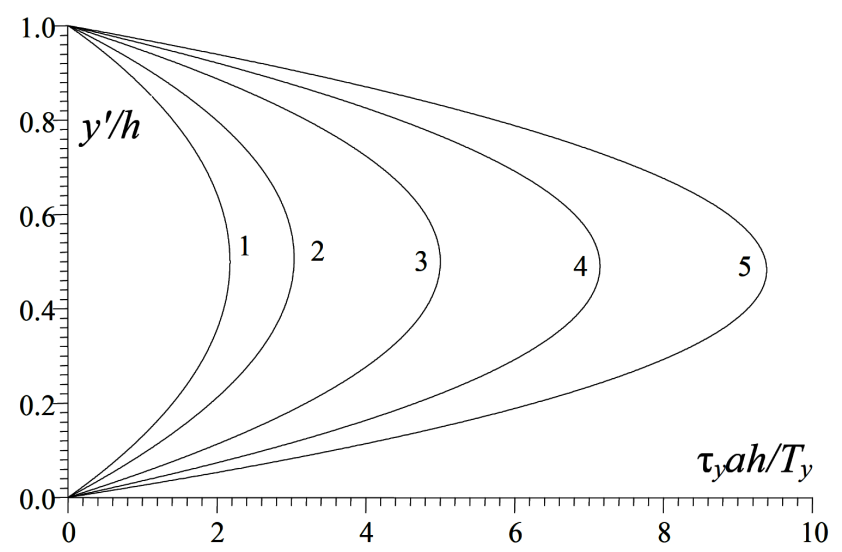

Fig. 7. Variation of the shear stress $\tau_{y} a h / T_{y}$ as a function of the ordinate $y^{\prime} / h$ for some sections according to the values of $n$ and $m$ of Table I.

the chosen sections when $T_{y}$ is applied. It also represents the variation of the length of the bandwidth $b_{y} / h$ as a function of the ordinate $x^{\prime} / a$ when $T_{x}$ is applied.

Figures 5 and 6 , respectively, represent the variation of the non-dimensional static moments $S_{x}^{*} /\left(a h^{2}\right)$ and $S_{y}^{*} /\left(a^{2} h\right)$ of the upper part of Fig. 2 according to the principle of Fig. 1 as a function of $y^{\prime} / h$, when a shear force $T_{y}$ is applied. We note that the static moments are zero at the high end $y^{\prime} / h=1.0$ because the upper part in this case does not exist and is also zero at the low end $y^{\prime} / h=0.0$ by what the static moments represent those of the complete section in relation to the central axes. Then the static moments take a maximum at a point in the section close to the centre of gravity.

Figure 7 shows the variation of the shear stress in the non-dimensional form as a function of the vertical ordinate $y^{\prime} / h$ when the shear force $T_{y}$ is applied. It is noted that the shear stress is zero at the upper and lower ends and takes a maximum at a point in the section which is not necessarily the centre of gravity. At the top and bottom ends, we have $S_{x}^{*}=0.0$ and $S_{y}^{*}=0.0$ from Figs. 5 and 6 and $b_{x}=0.0$ and $b_{y}=0.0$ from Fig. 2 . 


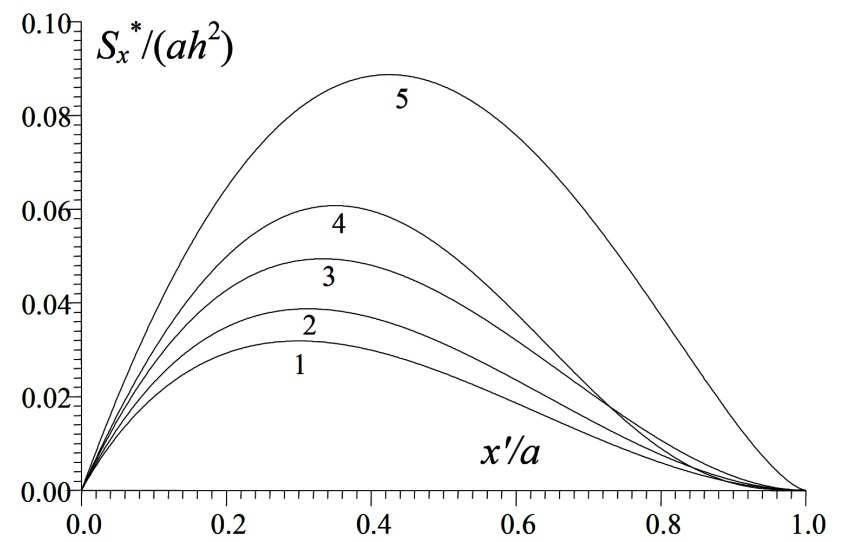

Fig. 8. Variation of the static moment $S_{x}^{*} /\left(a h^{2}\right)$ as a function of the ordinate $x^{\prime} / a$ for some sections according to the values of $n$ and $m$ of Table I.

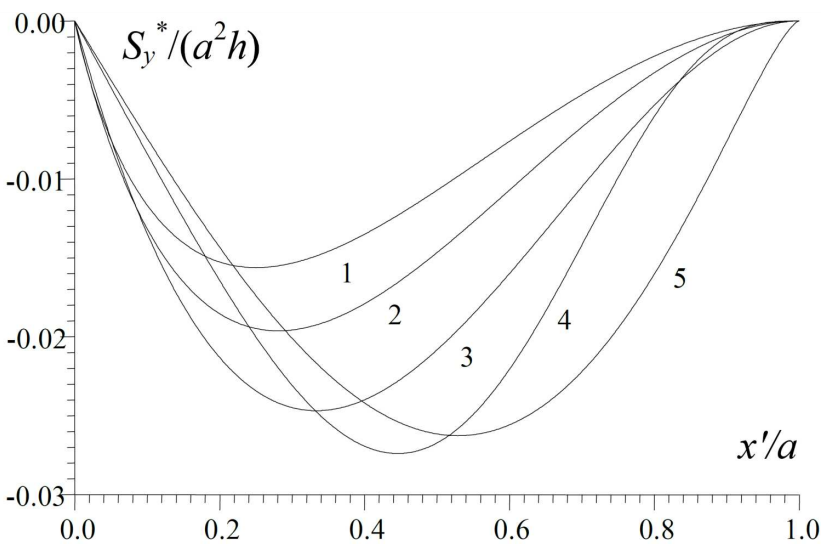

Fig. 9. Variation of the static moment $S_{y}^{*} /\left(a^{2} h\right)$ as a function of the ordinate $x^{\prime} / a$ for some sections according to the values of $n$ and $m$ of Table I.

Then, in accordance with relation (12) an indeterminacy of type $0 / 0$ is obtained. The value of the stress at this point cannot be computed numerically. However, we know physically that the stress is zero in these two points. Then the computation is approximated by the determination of the stress at the point $y^{\prime} / h=10^{-8}$ (close to zero) for the low part and at the point $y^{\prime} / h=1.0-10^{-8}$ close to one for the upper part.

Tables IV and V represent the numerical values of the geometrical characteristics of this first section. These values are computed numerically by our developed program by the use of the Gauss-Legendre quadrature of order 20. We note that the inertia product $I_{x y} \neq 0.0$. This demonstrates that the axis of application of the shear force is not a principal axis of inertia and that formula (13) is no longer valid in this case, where it makes the correction to this formula by the application of the relation (12).

In order to apply the relation (12), it is necessary to seek the orientation of the main axes of inertia and to consider the calculations according to these two axes after projecting the shear force $T_{y}$ along these two

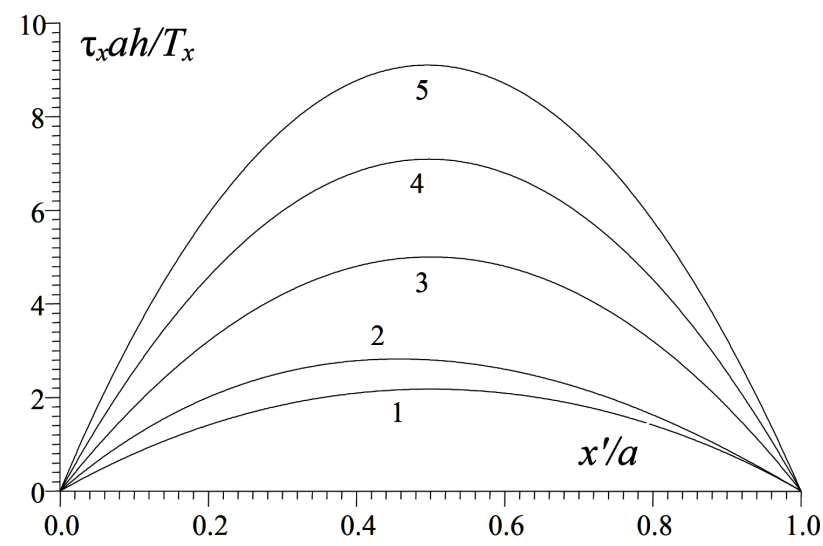

Fig. 10. Variation of the shear stress $\tau_{x} a h / T_{x}$ as a function of the ordinate $x^{\prime} / a$ for some sections according to the values of $n$ and $m$ of Table I.

TABLE IV

Area and centroid of some sections for selected values of $n$ and $m$ for the first section.

\begin{tabular}{c|c|c|c|c|c}
\hline \hline Curve & $n$ & $m$ & $A /(a h)$ & $x_{G}^{\prime} / a$ & $y_{G}^{\prime} / h$ \\
\hline 1 & $2 / 3$ & 1.0 & 0.40000 & 0.31250 & 0.28571 \\
2 & $1 / 2$ & 1.0 & 0.33333 & 0.30000 & 0.25000 \\
3 & 1.0 & 1.0 & 0.50000 & 0.33333 & 0.33333 \\
4 & 2.0 & 2.0 & 0.78540 & 0.42441 & 0.42441 \\
5 & 3.0 & 0.5 & 0.64285 & 0.35000 & 0.41538
\end{tabular}

axes. In this case the computational geometry becomes quite complicated where computation becomes almost impossible.

The numerical results found for the triangle $n=1.0$ and $m=1.0$ (curve 3 ) can be confirmed with the exact results given by the relationships (32), (33), and (34), which demonstrates the reliability of our numerical program.

Table VI represents the value and position of the maximum stress as well as the shape factor value $k_{y}$ for the five shapes chosen for the first section when the force $T_{y}$ is applied. The numerical results found for the triangle were confirmed when $n=m=1.0$ (curve 3 ) with relations (40) and (47).

For the application of the transverse force $T_{x}$, the variation of the static moments $S_{x}^{*}$ and $S_{y}^{*}$ is considered, as well as the shear stress respectively according to

TABLE V

Moments and product of inertia of some sections for selected values of $n$ and $m$ for the first section.

\begin{tabular}{c|c|c|c|c|c}
\hline \hline Curve & $n$ & $m$ & $I_{x} /\left(a h^{3}\right)$ & $I_{y} /\left(a^{3} h\right)$ & $I_{x y} /\left(a^{2} h^{2}\right)$ \\
\hline 1 & $2 / 3$ & 1.0 & 0.01814 & 0.02154 & -0.01071 \\
2 & $1 / 2$ & 1.0 & 0.01249 & 0.01761 & -0.00833 \\
3 & 1.0 & 1.0 & 0.02777 & 0.02777 & -0.01388 \\
4 & 2.0 & 2.0 & 0.05488 & 0.05488 & -0.01647 \\
5 & 3.0 & 0.5 & 0.04719 & 0.03236 & -0.01456
\end{tabular}


TABLE VI

Values of $\tau y_{\max }$, and $k_{y}$ of some sections for selected values of $n$ and $m$ for the first section.

\begin{tabular}{c|c|c|c|c|c}
\hline \hline Curve & $n$ & $m$ & $\tau y_{\max } /(a h)$ & $y^{\prime} / h$ & $k_{y}$ \\
\hline 1 & $2 / 3$ & 1.0 & 7.14637 & 0.49049 & 2.85854 \\
2 & $1 / 2$ & 1.0 & 9.38918 & 0.48148 & 3.12969 \\
3 & 1.0 & 1.0 & 5.00000 & 0.49949 & 2.50000 \\
4 & 2.0 & 2.0 & 2.17432 & 0.49949 & 1.70772 \\
5 & 3.0 & $1 / 2$ & 3.03597 & 0.50650 & 1.95169
\end{tabular}

TABLE VII

Values of $\tau x_{\max }$, and $k_{x}$ of some sections for selected values of $n$ and $m$ for the first section.

\begin{tabular}{c|c|c|c|c|c}
\hline \hline Curve & $n$ & $m$ & $\tau x_{\max } /(a h)$ & $x^{\prime} / a$ & $k_{x}$ \\
\hline 1 & $2 / 3$ & 1.0 & 7.08947 & 0.49749 & 2.83578 \\
2 & $1 / 2$ & 1.0 & 9.09962 & 0.49549 & 3.03317 \\
3 & 1.0 & 1.0 & 5.00000 & 0.49949 & 2.50000 \\
4 & 2.0 & 2.0 & 2.17423 & 0.49949 & 1.70765 \\
5 & 3.0 & $1 / 2$ & 2.81957 & 0.45645 & 1.81258
\end{tabular}

Figs. 8-10. The same comments presented for the case of the application of $T_{y}$ are valid for the case of $T_{x}$. The variations of $S_{x}^{*}$ and $S_{y}^{*}$ and $\tau_{x}$ are a function of $x^{\prime} / a$. They vary from left to right in this case. The functions are now null at the left and right ends.

Table VII represents the value and position of the maximum stress as well as the shape factor value $k_{x}$ for the five shapes chosen for the first section when the force $T_{x}$ is applied. The numerical results found for the triangle were confirmed when $n=m=1.0$ with relations (40) and (47).

\subsection{Second section}

Figures 11 to 16 contain 7 curves for 7 sections chosen according to the values of $\theta$. Then the curves 1 is for $\theta=20.0^{\circ}$. Curve 2 is for $\theta=30.0^{\circ}$. Curve 3 is for $\theta=45.0^{\circ}$. Curve 4 is for $\theta=60.0^{\circ}$. Curve 5 is for $\theta=70.0^{\circ}$. The curve 6 is for $\theta=80.0^{\circ}$ and the curve 7 is for $\theta=90.0^{\circ}$.

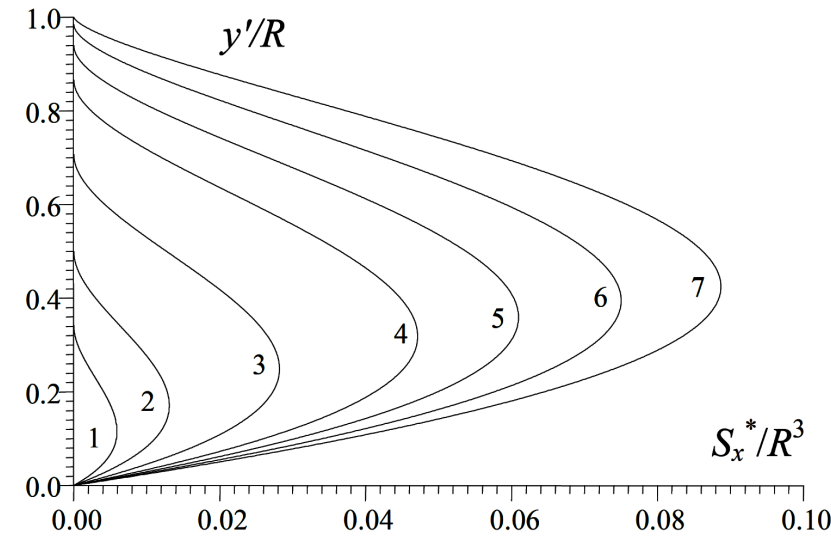

Fig. 11. Variation of static moment $S_{x}^{*} / R^{3}$ as a function of the ordinate $y^{\prime} / R$ for some sections.

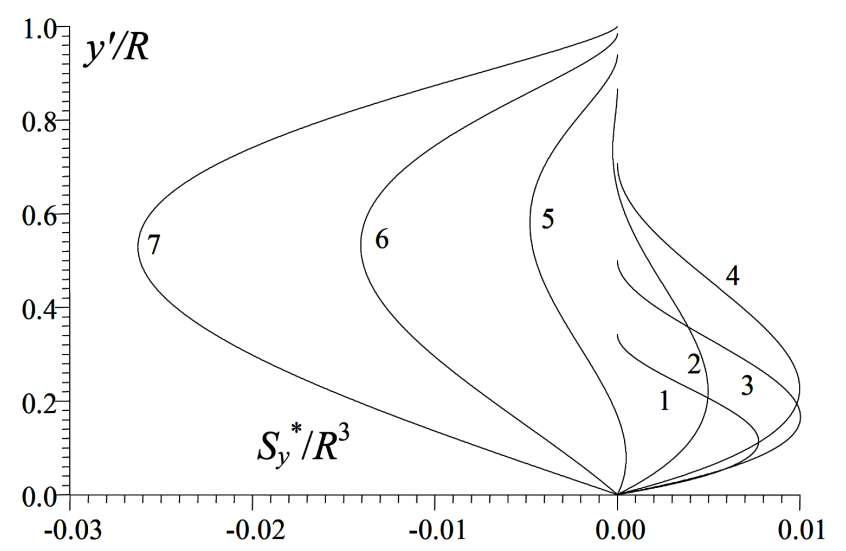

Fig. 12. Variation of static moment $S_{y}^{*} / R^{3}$ as a function of the ordinate $y^{\prime} / R$ for some sections.

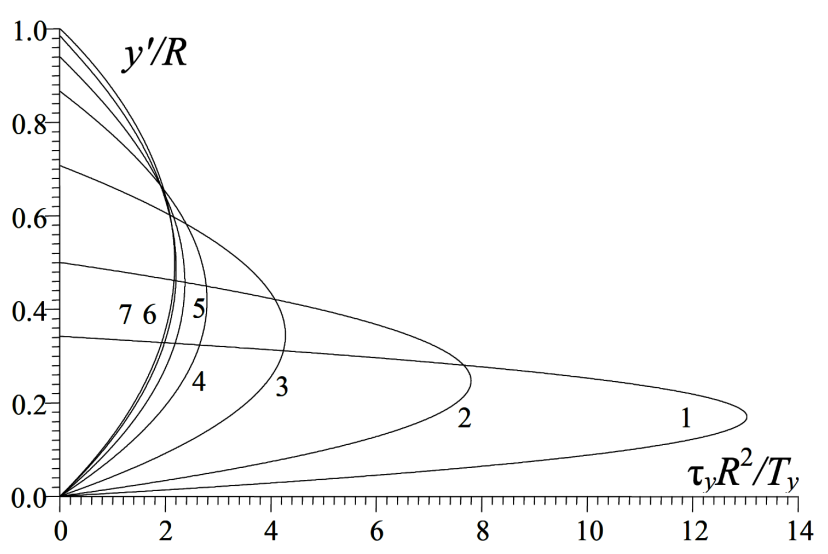

Fig. 13. Variation of the shear stress $\tau_{y} R^{2} / T_{y}$ as a function of the ordinate $y^{\prime} / R$ for some sections.

Figure 3 also shows the variation in the length of the bandwidth $b_{x} / R$ as a function of the $y^{\prime} / R$ ordinate for the selected sections when $T_{y}$ is applied. It also represents the variation of the length of the bandwidth $b_{y} / R$ as a function of the ordinate $x^{\prime} / R$ when $T_{x}$ is applied for the second section.

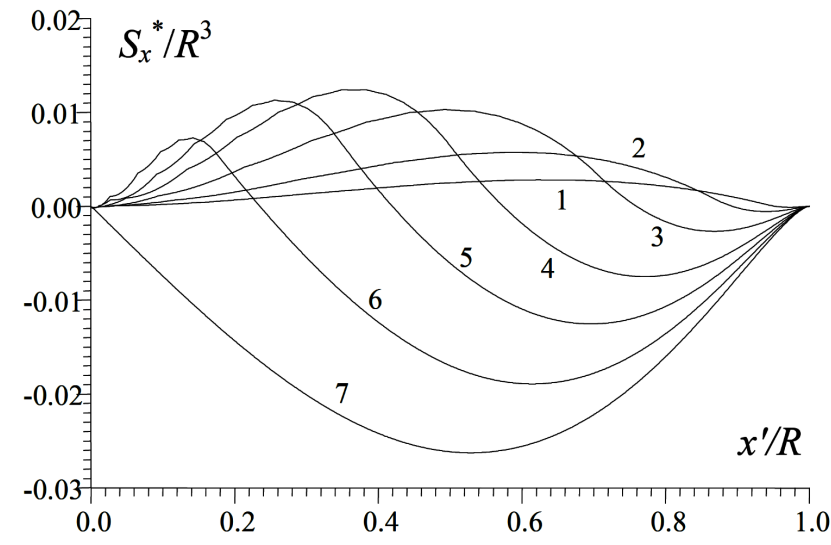

Fig. 14. Variation of static moment $S_{x}^{*} / R^{3}$ as a function of the ordinate $x^{\prime} / R$ for some sections. 


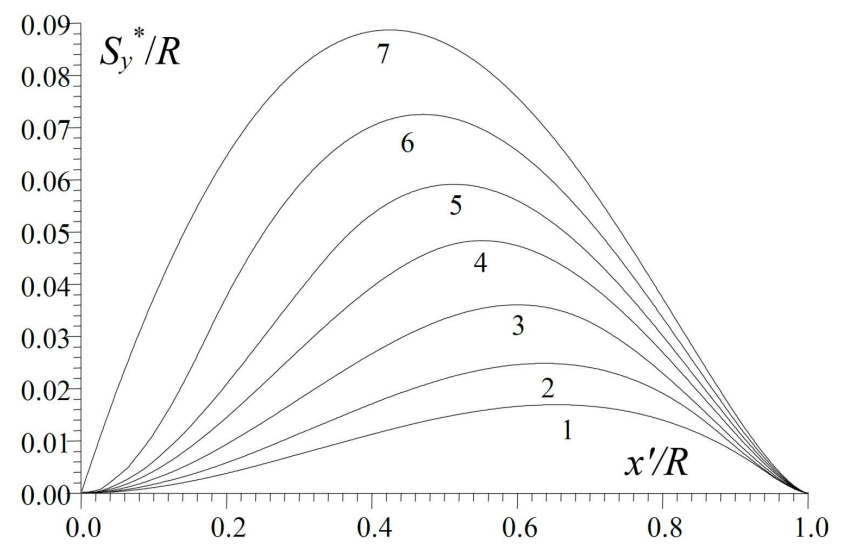

Fig. 15. Variation of static moment $S_{y}^{*} / R^{3}$ as a function of the ordinate $x^{\prime} / R$ for some sections.

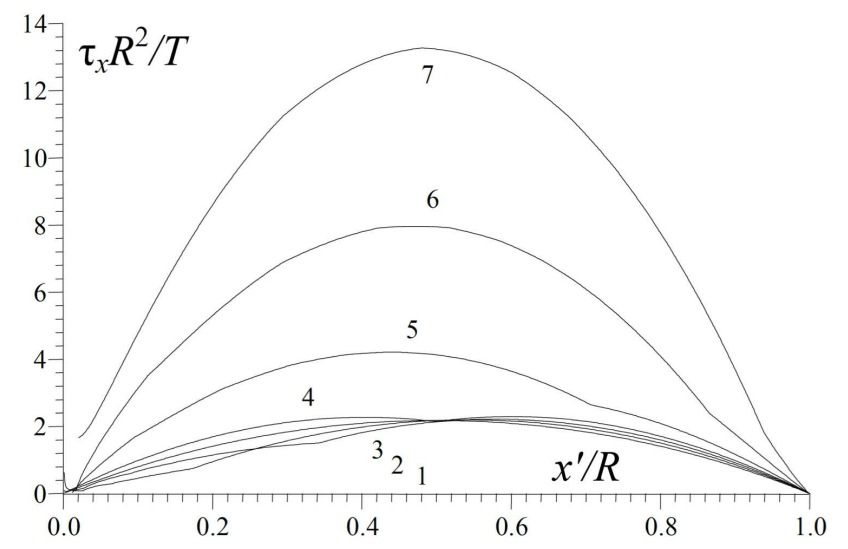

Fig. 16. Variation of the shear stress $\tau_{x} R^{2} / T_{x}$ as a function of the ordinate $x^{\prime} / R$ for some sections.

Figures 11 and 12 represent respectively the variation of the dimensionless static moments $S_{x}^{*} / R^{3}$ and $S_{y}^{*} / R^{3}$ of the upper part of Fig. 3 according to the principle of Fig. 1 as a function of $y^{\prime} / R$ when the shear force $T_{y}$ is applied.

Figure 13 shows the variation of the shear stress $\tau_{y} R^{2} / T_{y}$ in the non-dimensional form as a function of the vertical ordinate $y^{\prime} / R$ when the shear force $T_{y}$ is applied.

Tables VIII and IX represent the numerical values of the geometrical characteristics of this second section. These values are computed numerically by our developed program by the use of the Gauss-Legendre quadrature of order 20. We note that the inertia product $I_{x y} \neq 0.0$. This demonstrates that the axis of application of the shear force is not a principal axis of inertia and that formula (13) is no longer valid in this case, where it makes the correction to this formula by the application of the developed relation (12).

We can confirm the numerical results obtained for the quarter circle $\theta=90.0^{\circ}$ (curve 7 ) with the case of $n=$ 2.0 and $m=2.0$ (curve 4) for the first section, which demonstrates the reliability of our numerical program.
TABLE VIII

Area and centroid of some sections for selected values of $\theta$ for the second section.

\begin{tabular}{c|c|c|c|c}
\hline \hline Curve & $\theta\left[^{\circ}\right]$ & $A / R^{2}$ & $x_{G}^{\prime} / R$ & $y_{G}^{\prime} / R$ \\
\hline 1 & 20 & 0.17453 & 0.65321 & 0.11517 \\
2 & 30 & 0.26179 & 0.63661 & 0.17058 \\
3 & 45 & 0.39269 & 0.60021 & 0.24861 \\
4 & 60 & 0.52359 & 0.55132 & 0.31830 \\
5 & 70 & 0.61086 & 0.51276 & 0.35904 \\
6 & 80 & 0.69813 & 0.47021 & 0.39455 \\
7 & 90 & 0.78539 & 0.42441 & 0.42441
\end{tabular}

TABLE IX

Moments and product of inertia of some sections for selected values of $\theta$ for the second section.

\begin{tabular}{c|c|c|c|c}
\hline \hline Curve & $\theta\left[^{\circ}\right]$ & $I_{x} / R^{4}$ & $I_{y} / R^{4}$ & $I_{x y} / R^{4}$ \\
\hline 1 & 20 & 0.00114 & 0.00933 & 0.00149 \\
2 & 30 & 0.00370 & 0.01347 & 0.00281 \\
3 & 45 & 0.01140 & 0.01920 & 0.00390 \\
4 & 60 & 0.02372 & 0.02587 & 0.00186 \\
5 & 70 & 0.03379 & 0.03227 & -0.00208 \\
6 & 80 & 0.04447 & 0.04155 & -0.00828 \\
7 & 90 & 0.05487 & 0.05487 & -0.01647
\end{tabular}

Table $\mathrm{X}$ represents the value and the position of the maximum stress as well as the shape factor value $k_{y}$ for the seven shapes selected for the second section when the force $T_{y}$ is applied. We have confirmed the numerical results obtained for the quarter-circle case $\theta=90.0^{\circ}$ (curve 7 ) with case of $n=2.0$ and $m=2.0$ (curve 4 ) for the first section, which demonstrates the reliability of our numerical program.

For the application of the shear force $T_{x}$, we consider the variation of the static moments $S_{x}^{*}$ and $S_{y}^{*}$ as well as the shear stress, respectively, according to Figs. 14-16. The same comments presented for the case of the application of $T_{y}$ are valid for the case of $T_{x}$. The variation of $S_{x}^{*}$ and $S_{y}^{*}$ and $\tau_{x}$ depend on $x^{\prime} / R$. They vary from left to right in this case. The functions are now null at the left and right ends.

Table XI represents the value and position of the maximum stress as well as the shape factor value $k_{x}$ for the five shapes chosen for the first section when the force $T_{x}$

TABLE X

Values of $\tau y_{\max }$, and $k_{y}$ of some sections for selected values of $\theta$ for the second section.

\begin{tabular}{c|c|c|c|c}
\hline \hline Curve & $\theta\left[^{\circ}\right]$ & $\tau y_{\max } / R^{2}$ & $y^{\prime} / R$ & $k_{y}$ \\
\hline 1 & 20 & 13.03241 & 0.17015 & 2.27458 \\
2 & 30 & 7.79483 & 0.24674 & 2.04068 \\
3 & 45 & 4.27495 & 0.34470 & 1.67877 \\
4 & 60 & 2.78723 & 0.41957 & 1.45939 \\
5 & 70 & 2.36675 & 0.45620 & 1.44576 \\
6 & 80 & 2.20383 & 0.48402 & 1.53856 \\
7 & 90 & 2.17430 & 0.49949 & 1.70769
\end{tabular}


is applied. We confirmed the numerical results found for the $\theta=90.0^{\circ}$ (curve 7 ) with the case of $n=2.0$ and $m=2.0$ (curve 4) for the first section, which demonstrates the reliability of our numerical program.

TABLE XI

Values of $\tau x_{\max }$, and $k_{x}$ of some sections for selected values of $\theta$ for the second section.

\begin{tabular}{c|c|c|c|c}
\hline \hline Curve & $\theta\left[^{\circ}\right]$ & $\tau x_{\max } / R^{2}$ & $x^{\prime} / R$ & $k_{x}$ \\
\hline 1 & 20 & 13.26207 & 0.48048 & 2.31462 \\
2 & 30 & 7.96378 & 0.47147 & 2.08483 \\
3 & 45 & 4.21175 & 0.44244 & 1.65391 \\
4 & 60 & 2.29435 & 0.60059 & 1.20132 \\
5 & 70 & 2.20977 & 0.57757 & 1.34987 \\
6 & 80 & 2.19126 & 0.54254 & 1.52979 \\
7 & 90 & 2.17439 & 0.49949 & 1.70776
\end{tabular}

\subsection{Third section}

Figures 17 to 22 contain 5 curves for 5 sections chosen according to the values of $n$ of Table II. Then curve 1 is for $n=1 / 2$. Curve 2 is for $n=2 / 3$. Curve 3 is for $n=1.0$. Curve 4 is for $n=2.0$. Curve 5 is for $n=3.0$.

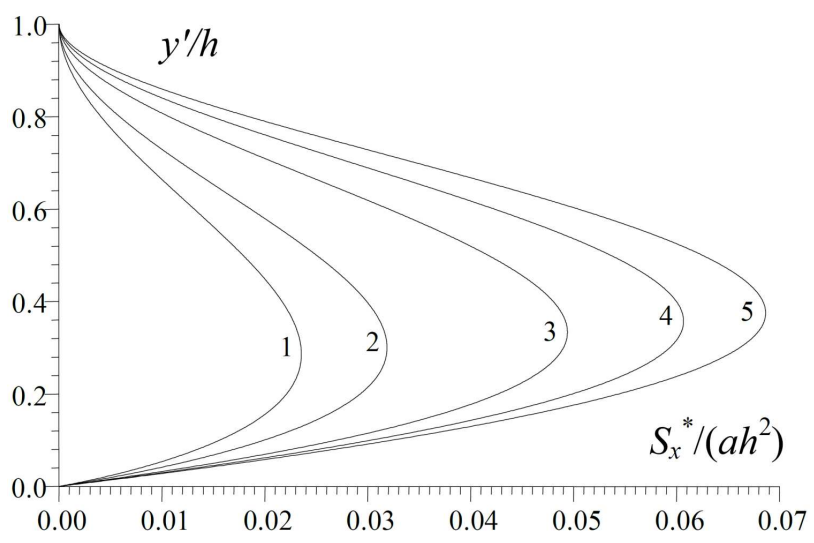

Fig. 17. Variation of static moment $S_{x}^{*} /\left(a h^{2}\right)$ as a function of the ordinate $y^{\prime} / h$ for some sections according to the values of $n$ of Table II.

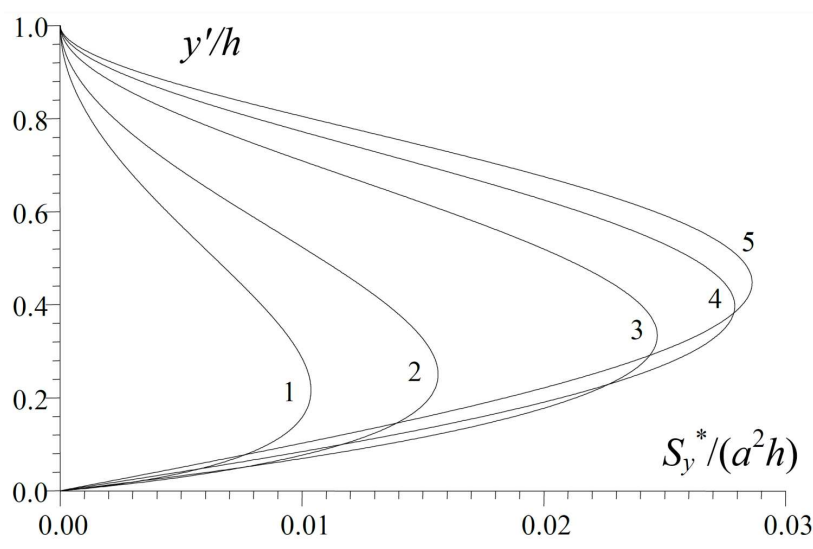

Fig. 18. Variation of static moment $S_{y}^{*} /\left(a^{2} h\right)$ as a function of the ordinate $y^{\prime} / h$ for some sections according to the values of $n$ of Table II.

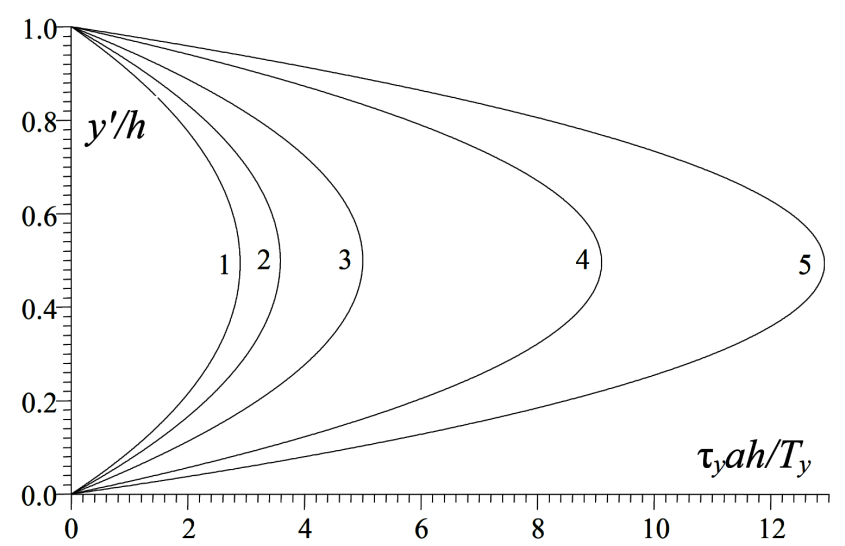

Fig. 19. Variation of the shear stress $\tau_{y} a h / T_{y}$ as a function of the ordinate $y^{\prime} / h$ for some sections according to the values of $n$ of Table II.

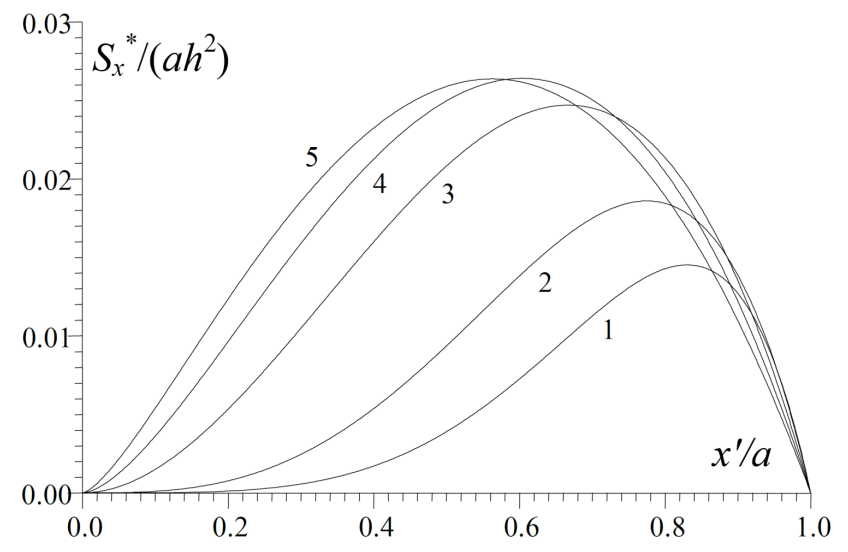

Fig. 20. Variation of static moment $S_{x}^{*} /\left(a h^{2}\right)$ as a function of the ordinate $x^{\prime} / a$ for some sections according to the values of $n$ of Table II.

Figure 4 shows the variation in the length of the bandwidth $b_{x} / a$ as a function of the ordinate $y^{\prime} / h$ for the sections chosen when $T_{y}$ is applied. It also represents the variation in the length of the bandwidth $b_{y} / h$ as a function of the ordinate $x^{\prime} / a$ when $T_{x}$ is applied for the second section.

Figures 17 and 18 represent, respectively, the variation of the dimensionless static moments $S_{x}^{*} /\left(a h^{2}\right)$ and $S_{y}^{*} /\left(a^{2} h\right)$ of the upper part of Fig. 4 according to the principle of Fig. 1 as a function of $y^{\prime} / h$ when the shear force $T_{y}$ is applied.

Figure 19 shows the variation of the shear stress in the non-dimensional form as a function of the vertical ordinate $y^{\prime} / h$ when the shear force $T_{y}$ is applied. It is noted that the shear stress is zero at the upper and lower ends and takes a maximum at a point in the section which is not necessarily the centre of gravity.

Tables XII and XIII represent the numerical values of the geometrical characteristics of this third section. These values are computed numerically by our developed program by the use of the Gauss-Legendre quadrature of order 20 . We note that the inertia product $I_{x y} \neq 0.0$. 


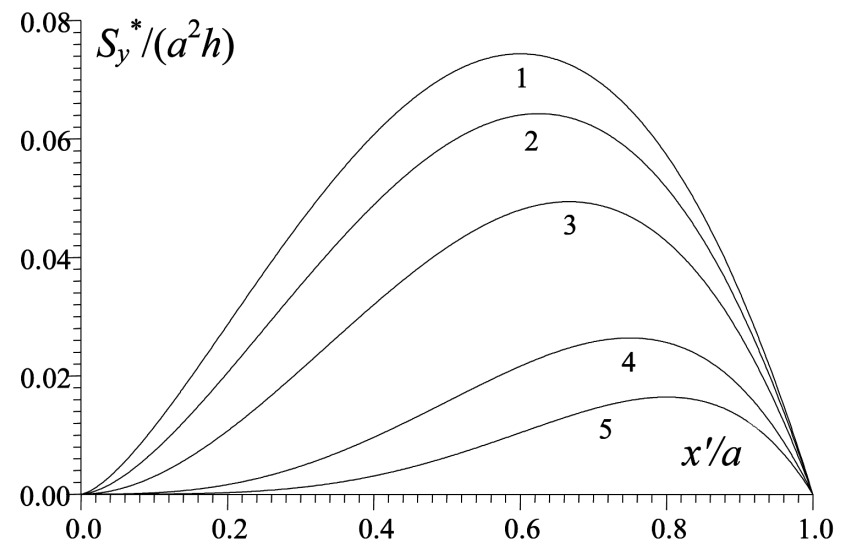

Fig. 21. Variation of static moment $S_{y}^{*} /\left(a^{2} h\right)$ as a function of the ordinate $x^{\prime} / a$ for some sections according to the values of $n$ of Table II.

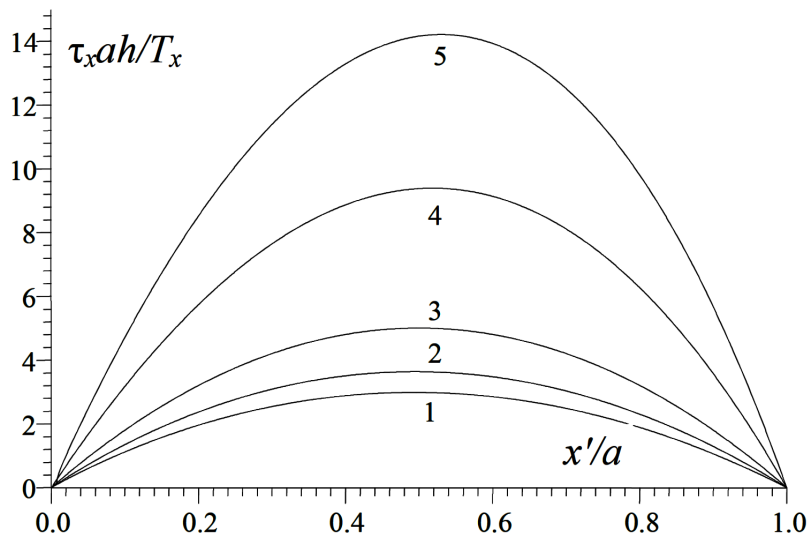

Fig. 22. Variation of tangential stress $\tau_{x} a h / T_{x}$ as a function of the ordinate $x^{\prime} / a$ for some sections according to the values of $n$ of Table II.

This demonstrates that the axis of application of the shear force is not a principal axis of inertia and that formula (13) is no longer valid in this case, where it makes the correction to this formula by the application of the developed relation (12).

We can confirm the numerical results found for triangle $n=1.0$ (curve 3 ) with the exact results given by relations (32), (33), and (34) and with the triangle results for $n=m=1.0$ for first section (curve 3), which demonstrates the reliability of our digital program.

\section{TABLE XII}

Area and centroid of some sections for selected values of $n$ for the third section.

\begin{tabular}{c|c|c|c|c}
\hline \hline Curve & $n$ & $A /(a h)$ & $x_{G}^{\prime} / a$ & $y_{G}^{\prime} / h$ \\
\hline 1 & $1 / 2$ & 0.66666 & 0.60000 & 0.37500 \\
2 & $2 / 3$ & 0.60000 & 0.62500 & 0.35714 \\
3 & 1.0 & 0.50000 & 0.66666 & 0.33333 \\
4 & 2.0 & 0.33333 & 0.75000 & 0.30000 \\
5 & 3.0 & 0.25000 & 0.80000 & 0.28571
\end{tabular}

TABLE XIII

Moments and product of inertia of some sections for selected values of $n$ for the third section.

\begin{tabular}{c|c|c|c|c}
\hline \hline Curve & $n$ & $I_{x} /\left(a h^{3}\right)$ & $I_{y} /\left(a^{3} h\right)$ & $I_{x y} /\left(a^{2} h^{2}\right)$ \\
\hline 1 & $1 / 2$ & 0.03958 & 0.04571 & 0.01666 \\
2 & $2 / 3$ & 0.03458 & 0.03835 & 0.01607 \\
3 & 1.0 & 0.02777 & 0.02777 & 0.01388 \\
4 & 2.0 & 0.01761 & 0.01250 & 0.00833 \\
5 & 3.0 & 0.01292 & 0.00666 & 0.00535
\end{tabular}

Table XIV represents the value and position of the maximum stress as well as the shape factor value $k_{y}$ for the five shapes chosen for the first section when the force $T_{y}$ is applied.

TABLE XIV

Values of $\tau y_{\max }$, and $k_{y}$ of some sections for selected values of $n$ for the third section.

\begin{tabular}{c|c|c|c|c}
\hline \hline Curve & $n$ & $\tau y_{\max } /(a h)$ & $y^{\prime} / h$ & $k_{y}$ \\
\hline 1 & $1 / 2$ & 2.89552 & 0.49349 & 1.93035 \\
2 & $2 / 3$ & 3.58408 & 0.49849 & 2.15044 \\
3 & 1.0 & 5.00000 & 0.49949 & 2.50000 \\
4 & 2.0 & 9.09956 & 0.49549 & 3.03318 \\
5 & 3.0 & 12.92857 & 0.49249 & 3.23214
\end{tabular}

TABLE XV

Values of $\tau x_{\max }$, and $k_{x}$ of some sections for selected values of $n$ for the third section.

\begin{tabular}{c|c|c|c|c}
\hline \hline Curve & $n$ & $\tau x_{\max } /(a h)$ & $x^{\prime} / a$ & $k_{x}$ \\
\hline 1 & $1 / 2$ & 2.98313 & 0.49349 & 1.98875 \\
2 & $2 / 3$ & 3.63274 & 0.49449 & 2.17964 \\
3 & 1.0 & 5.00000 & 0.49949 & 2.50000 \\
4 & 2.0 & 9.38768 & 0.51851 & 3.12922 \\
5 & 3.0 & 14.20847 & 0.52952 & 3.55211
\end{tabular}

For the application of the shear force $T_{x}$, we consider the variation of the static moments $S_{x}^{*}$ and $S_{y}^{*}$ as well as the shear stress $\tau_{x} a h / T_{x}$, respectively, according to Figs. 20-22. The same comments presented for the case of the application of $T_{y}$ are valid for the case of $T_{x}$. The variations of $S_{x}^{*}$ and $S_{y}^{*}$ and $\tau_{x}$ are a function of $x^{\prime} / a$. They vary from left to right in this case. The functions are now null to the left and right ends.

Table XV represents the value and position of the maximum stress as well as the shape factor value $k_{x}$ for the five shapes chosen for the third section when the force $T_{x}$ is applied. The numerical results found for the triangle when $n=1.0$ were confirmed with relations (40) and (47) and with the triangle results for the case $n=m=1.0$ (curve 3) for the first section.

Finally, a criterion of resistance of the beams of moderate lengths subjected to bending can be applied to avoid breaking the beam by [1-3]: 


$$
\sqrt{\sigma_{\text {Max }}^{2}+4 \tau_{\text {Max }}^{2}}<\sigma_{\text {eleg }} .
$$

In relation (86), we clearly notice the influence of $\tau_{\text {Max }}$ in the balance of applied stresses, which is accompanied by the stress $\sigma_{\mathrm{Max}}$ due to the bending moment.

The application of this criterion will determine the size of the section with a high precision without breaking. That is, the dimension of the section with consideration of $\tau_{\mathrm{Max}}$ is less than the dimension of the no-effect section of $\tau_{\mathrm{Max}}$.

\section{Conclusion}

This work allows us to calculate the shear stress due to the effect of shear force for general case of deflected bending theory in arbitrary complex unsymmetrical sections for the assumptions and constraints given in our mathematical formulation. We can draw the following conclusions:

1. The numerical program can make any unsymmetrical sections.

2. Each section has two shape factors $k_{x}$ and $k_{y}$ following the two options of two shear forces $T_{x}$ and $T_{y}$.

3. For $T=T_{y}$, the stress $\tau$ will be forced to zero at the top and bottom ends and is zero at the left and right ends if $T=T_{x}$.

4. When $T$ is directed arbitrarily in the plane of the section, it must be the projection as the horizontal and vertical axes which cannot be forced as a principal axis of inertia, and we consider the calculation of the stress $\tau$ along the two axes and make the vector summation.

5. The stress $\tau_{\text {Max }}$ is at a point which is not the section centre of gravity.

6. The calculation can be made to the two arbitrary axes.

7. The calculation of the geometric characteristics of the section is necessary.

8. The value of $T$ in the program does not matter. However, we calculate the value of $\tau / T$ as a function of the geometry of the section.

9. The calculation is done in a non-dimensional way. However, the values of $a, h$ and $R$ of the selected sections have no significant values.

10. The calculation of the geometrical characteristics of the section as well as the static moments of the upper and right parts is done by a numerical integration of the functions which depend on the shape of the chosen section.
11. The numerical integration of the functions found is done by the quadrature of Gauss-Legendre of order $q=20$ designed for the regular functions given its rapidity of convergence and high precision.

12. The maximum stress in all cases is always higher than the average stress. In other words, the form factor is always greater than unity.

13. The distribution of $\tau$ and the value of the maximum stress $\tau_{\text {Max }}$ as well as the shape factor depends on the orientation of the central axis. The smallest value of the maximum stress is along the principal axis of inertia and the greatest value is also along the other principal axis of inertia.

14. The maximum stress and the shape factor depends on the rotation of the central axis in the plane of the section.

As perspective and in the same line of research, we can consider the calculation of the shear stress due to shear forces for arbitrary unsymmetrical thin-walled hollow sections.

\section{Acknowledgments}

The author acknowledges Khaoula Yahiaoui, Abdelghani Amine Yahiaoui, Ritadj Yahiaoui and Assil Yahiaoui without forgot Mouza Ouahiba for granting time to prepare this manuscript.

\section{References}

[1] S. Timoshenko, J. Goodier, Theory of Elasticity, McGraw-Hill Book Co., 1970.

[2] F.P. Beer, Mechanics of Materials, 7th ed., McGrawHill Education Book Co., 2015.

[3] S. Timoshenko, History of Strength of Materials McGraw-Hill Book Co., 1983.

[4] S. Ramamrutham, R. Narayanan, Strength of Materials 14th ed., Dhanpat Rai Publ., 2011.

[5] D. Petru, Mech. Eng. 17, 39 (2012).

[6] S. Nair, E. Reissner, J. Appl. Mech. 43, 75 (1976).

[7] R. Barretta, A. Barretta, Europ. J. Mech. A Solids 29, 400 (2010).

[8] G.R. Cowper, J. Appl. Mech. 33, 335 (1966).

[9] J. Brnic, G. Turkalj, M. Canadija, Meccanica 45, 227 (2010).

[10] A. Kirad, T. Zebbiche, M. Boun-jad, Mech. Industry 17, 608 (2016).

[11] E. Velazquez, J.B. Kosmatka, J. Appl. Mech. 80 , 011010 (2013).

[12] J.M. Segura, G. Armengaud, Arch. Appl. Mech. 68, 206 (1998).

[13] E.J. Sapountzakis, D.G. Panagos, Arch. Appl. Mech. 78, 687 (2008).

[14] S.I. Suzuki, AIAA J. 24, 1396 (1986). 
[15] A. Steinboeck, A. Kugi, H.A. Mang, Int. J. Solids Struct. 50, 1859 (2013).

[16] A. Ralston, P. Rabinowitz, A First Course in Numerical Analysis, McGraw-Hill Book Co., 1985.

[17] B. Demidovitch, I. Marron, Elements of Numerical Calculation, Edition Mir, Moscow 1987 (in French).

[18] G.H. Golub, J.H. Welsch, Math. Computat. 23, 221 (1969).

[19] D. Kahaner, C. Moler, S. Nash, Numerical Methods and Software, Prentice-Hall, 1989.
[20] D.P. Laurie, J. Comput. Appl. Math. 112, 165 (1999).

[21] D.P. Laurie, J. Comput. Appl. Math. 127, 201 (2001).

[22] P.N. Swarztrauber, SIAM J. Sci. Comput. 24, 945 (2003).

[23] H. Wang, S. Xiang, Math. Comp. 81, 861 (2012). 